\title{
Global gene expression profiles for the growth phases of Trichophyton rubrum
}

\author{
XU Xing Ye ${ }^{1}$, LIU Tao ${ }^{2}$, LENG WenChuan ${ }^{2}$, DONG Jie $^{2}$, XUE Ying ${ }^{2}$, \\ YANG HanChun ${ }^{1 *} \& \mathrm{JIN}^{2} \mathrm{ii}^{* *}$ \\ ${ }^{1}$ Key Laboratory of Animal Epidemiology and Zoonosis, Ministry of Agriculture, and State Key Laboratory of Agrobiotechnology, \\ College of Veterinary Medicine, China Agricultural University, Beijing 100094, China; \\ ${ }^{2}$ State Key Laboratory for Molecular Virology and Genetic Engineering, Institute of Pathogen Biology, \\ Chinese Academy of Medical Sciences, Beijing 100176, China
}

Received February 15, 2011; accepted May 26, 2011; published online June 11, 2011

\begin{abstract}
Trichophyton rubrum (T. rubrum) is a common superficial fungus. Molecular and genetic studies of T. rubrum are still limited. In this paper, we report the global analysis of gene expression profiles at different growth phases using cDNA microarray technology. A total of 2044 differentially expressed genes were obtained and clustered into three expression patterns. Our data confirmed previous results that many mRNAs were pre-stored in the conidia of T. rubrum. Transcriptional profiling and function analysis showed that some glycolytic enzymes share similar expression patterns and may be coregulated during the transition of growth phases. Some genes involved in small GTPase signaling pathways, and in cAMP-dependent and MAPK regulation pathways were induced in response to the growth dynamics of T. rubrum. Although the detailed biological roles of these $T$. rubrum genes are still unknown, our results suggest that these genes may be involved in regulation mechanisms in the life cycle of the fungus.
\end{abstract}

Trichophyton rubrum, gene expression profiles, cDNA microarray, growth phases

Citation: $\quad$ Xu X Y, Liu T, Leng W C, et al. Global gene expression profiles for the growth phases of Trichophyton rubrum. Sci China Life Sci, 2011, 54: 675-682, doi: 10.1007/s11427-011-4187-5

The dimorphic fungus Trichophyton rubrum (T. rubrum) is a worldwide pathogen that causes various superficial infections, such as tinea capitis, tinea corporis, tinea inguinalis, tinea manus, tinea unguium and tinea pedis [1-3]. Unlike most other medically important fungi that are opportunists, dermatophytes are highly specialized fungi that are obligate pathogens [4,5]. Moreover, T. rubrum infections are often intractable, and relapse frequently occurs after the cessation of antifungal therapy [6,7].

Microarray technology is a powerful tool to characterize gene functions and discover the functionally related genes needed for developmental and behavioral processes [8,9]. Microarray studies have examined global gene expression

*Corresponding author (email: yanghanchun1@ cau.edu.cn; zdsys@ vip.sina.com) in over 20 species of filamentous fungi encompassing a wide variety of research areas [10].

The prevalence of infections caused by T. rubrum and its anthrophilic nature make it a good model for the study of human pathogenic filamentous fungi. To carry out a comprehensive investigation, we constructed cDNA libraries for different stages of T. rubrum. 11085 unique ESTs sequences from the cDNA libraries were obtained and annotated. These sequences represent $85 \%$ of the predicated genes [11,12]. Using complementary DNA microarray technology, we analyzed changes in gene expression during conidia germination and reported the effects of several antifungal agents on the gene expression profile of T. rubrum [13-15]. In the present study, we investigated the growth dynamics and characterized the changes of gene expression that take place 
during the period when T. rubrum was cultured in Sabouraud liquid medium. The results reveal the gene expression patterns at different growth stages of T. rubrum and give a clue for better understanding of the genetic characteristics and development of the fungus.

\section{Materials and methods}

\subsection{Fungal strain}

T. rubrum (strain BMU01672) selected for this study was isolated from nail scraps of a patient suffering from tinea unguium. The strain was provided by Professor Li RuoYu (Research Center for Medical Mycology, Peking University, Beijing, China). The strain was confirmed as T. rubrum by morphologic identification and by PCR amplification and sequencing of the $18 \mathrm{~S}$ ribosomal DNA and ITS regions.

The BMU01672 isolate was cultured on potato glucose agar (Difco) at $28^{\circ} \mathrm{C}$ for $10-15 \mathrm{~d}$ to produce conidia. Conidia were collected from the agar and transferred into sterile double-distilled water. After filtering twice through a $70 \mu \mathrm{m}$ nylon filter, the suspension was adjusted to a concentration of $5 \times 10^{7}-8 \times 10^{7}$ conidia $\mathrm{mL}^{-1}$ for use in the following study.

\subsection{Cell culture for microarray experiments}

To determine the time points for the analysis of the transcriptional profile at different growth stages, ten $250 \mathrm{~mL}$ flasks containing $100 \mathrm{~mL}$ of Sabouraud liquid medium (containing $49 \mathrm{~g}$ of glucose and $10 \mathrm{~g}$ of Difco Bacto-peptone in $1 \mathrm{~L}$ distilled water) were inoculated with $5 \times 10^{5}$ conidia $\mathrm{mL}^{-1}$ and cultured with constant shaking at $200 \mathrm{r} \mathrm{min}^{-1}$ in an Innova 4230 refrigerated incubator shaker (New Brunswick Scientific, Edison, NJ, USA) at $28^{\circ} \mathrm{C}$. After every $24 \mathrm{~h}, T$. rubrum was collected from one of the flasks and the dry mass was weighed to monitor the growth dynamics of T. rubrum.

For the microarray experiments, another nine $250 \mathrm{~mL}$ flasks, containing $100 \mathrm{~mL}$ of Sabouraud liquid medium and $5 \times 10^{5}$ conidia $\mathrm{mL}^{-1}$ were incubated using the same culture conditions described above. Three time points, 2, 6 and 10 days after cultivation, were selected to investigate transcriptional changes. At each of the time points, three flasks of mycelia were independently harvested as replications, frozen in liquid nitrogen, ground to powder, and used for RNA preparation. Similarly, the stock conidia were used as the time point 0 (zero) samples.

\section{3 cDNA microarray construction}

The PCR fragments used for printing the microarray chip were amplified from the T. rubrum EST library with T7 and SP6 universal primers. For each unique EST, a gene-spe- cific 18-mer oligonucleotide was synthesized and used to PCR-amplify specific DNA fragments. To produce the microarrays, the PCR products were subsequently purified using MultiScreen-PCR plates (Millipore) and resuspended in $12 \mu \mathrm{L}$ of spotting solution containing $50 \%$ dimethyl sulfoxide. A set of microarrays containing a total of 11232 spots (10250 clones in the form of PCR products and 982 controls that included blank, negative, and positive controls) were spotted in duplicate on $\beta$ amino propylsilan coated GAP II slides (Corning) with a Cartesian ${ }^{\circledR}$ arrayer. The spotted cDNA was cross-linked to the surface of the slides (at $65 \mathrm{~mJ}$ ) using a StrataLinker instrument (Stratagene) and washed with $1 \%$ SDS to minimize the background. All GenBank IDs, contigs assembly information, and EST annotations used in the cDNA microarray can be obtained from the T. rubrum database (http://www.mgc.ac.cn/TrED/) [16].

\subsection{RNA preparation}

To disrupt the cells, 12 frozen T. rubrum BMU 01672 samples from 0 to $10 \mathrm{~d}$ were separately ground to powder in liquid nitrogen using a mortar and pestle. Total RNA was isolated from each sample using the Qiagen RNeasy ${ }^{\circledR}$ Plant Mini Kit (Qiagen, Inc., Valencia, CA) according to the manufacturer's instructions. The RNA concentration and purity were determined spectrophotometrically by measuring absorbance at 230, 260, 280, and $320 \mathrm{~nm}$. Purity and integrity of the RNA were confirmed by agarose gel electrophoresis. Poly $(\mathrm{A})^{+}$mRNA was isolated with the Oligotex mRNA Mini Kit (Qiagen).

\subsection{Microarray hybridization}

mRNA from $20 \mu \mathrm{g}$ of each of the total RNA samples was reversely transcribed into cDNA and labeled with the fluorescent dye Cy5. A reference genomic DNA fragment $(5 \mu \mathrm{g})$ isolated in the hyphal stage was labeled with $\mathrm{Cy} 3$ as previous report [13].

The labeled cDNAs and the reference DNA were purified using the QIAquick PCR Purification Kit (Qiagen), then mixed and resuspended in $10 \mu \mathrm{L}$ of distilled water to which $1.5 \mu \mathrm{L}$ of $50 \times$ Denhardt's solution, $2.25 \mu \mathrm{L}$ of $20 \times$ SSC, $1.125 \mu \mathrm{L}$ of $500 \mu \mathrm{g} \mathrm{mL}^{-1}$ yeast tRNA, $0.375 \mu \mathrm{L}$ of $1 \mathrm{~mol} \mathrm{~L}{ }^{-1}$ HEPES (pH 7.0), $0.375 \mu \mathrm{L}$ of $10 \%$ SDS, $2 \mu \mathrm{L}$ of poly(A) $\left(5 \mathrm{mg} \mu \mathrm{L}^{-1}\right)$ were added. The mixture was heated at $100^{\circ} \mathrm{C}$ for $3 \mathrm{~min}$. Hybridization was carried out as described by Hayes et al. [17].

\subsection{Data analysis}

Images of the microarrays were acquired using a GenePix 4000A microarray scanner (Axon Instruments, USA) and the spots were quantified using GenePix Pro 6.0 software. 
Bad spots were flagged automatically by the software and each slide was subsequently inspected manually. The multiple data sets that fitted all the following features were flagged: spot diameter $\geqslant 80 \mu \mathrm{m}, \% \mathrm{~B}$ (532 or 635) and $2 \mathrm{SD}>55$, SNR635 (or 532) $\geqslant 3$. These data sets were normalized (the ratio of medians of all features equals one) using the GenePix Pro software (version 6.0) and then further normalized in two steps: total intensity and Lowess normalization using Tiger MIDAS V2.19 [18]. After normalization, the expression data for every gene was subjected to one way ANOVA $(P<0.01)$ using the Tiger TMEV 3.1 software to identify the genes for which the expression levels were dramatically altered during different growth stages [18]. The changes in expression levels of each significant gene were also compared to the expression levels at time point 0 and the 2044 genes for which changes in expression were more than two-fold during the process were used for further analysis.

\subsection{Validation of microarray data through real-time RT-PCR}

Aliquots of the RNA preparations from each of the time point samples used in the microarray experiments were saved for quantitative real-time RT-PCR. Eight of the genes predicted to be differentially expressed by microarray analysis were tested by quantitative reverse transcription (RT)-PCR with an Applied Biosystems (Foster City, CA, USA) GeneAmp7000 sequence detection system. Genespecific primers were designed using Primer Express software (Applied Biosystems) and the sequences are shown in Table 1. 18S ribosomal cDNA was used as a control reference. The changes in fluorescence of SYBR Green I dye

Table 1 Sequences of the primers used in the real-time RT-PCR assays

\begin{tabular}{cl}
\hline Target genes & \multicolumn{1}{c}{ Primer sequence $^{\text {a) }}$} \\
\hline \multirow{2}{*}{ 18S } & F, 5'-CGCTGGCTTCTTAGAGGGACTAT-3' \\
& R, 5'-TGCCTCAAACTTCCATCGACTT-3' \\
DW685106 & F, 5'-CCTGCTTGATGGTGGAAA-3' \\
& R, 5'-GGTCTCGGTGGAGGTAAAA-3' \\
DW680891 & F, 5'-TTCATCGCTTCAAAGTCATCC-3' \\
& F, 5'-ACGGCTCTTATACCAGGGTG-3' \\
DW679448 & F, 5'-TGACACCGAACTATGGAGC-3' \\
& R, 5'-TGCTTCATCAAGCACCAAAATGTTG-3' \\
DW678242 & F, 5'-TGGACTGCTTCAGCGACAA-3' \\
& R, 5'-ATCGGTGAGCGAAATGGT-3' \\
DW678984 & F, 5'-CCTTCTACGGAGGCAGTT-3' \\
& R, 5'-CAGACGAAAGCAGGCAAA-3' \\
DW681520 & F, 5'-AGGACGAGCAATCATACATC-3' \\
& R, 5'-CCGCTTGAGCCACCATAC-3' \\
DW679821 & F, 5'-GAGGTGTTTATCTTTTCGCTGTC-3' \\
& R, 5'-AGGTTTGTATTTGGGGTATCC-3' \\
& F, 5'-AACCTGACGAGCAAACCAA-3' \\
DW691154 & R, 5'-AATGACAACAGAGGCGATAAAG-3'
\end{tabular}

a) $\mathrm{F}$, forward primer; $\mathrm{R}$, reverse primer. were monitored by the GeneAmp7000 software, and the threshold cycle $\left(C_{\mathrm{T}}\right)$ above the background for each reaction was calculated. The $C_{\mathrm{T}}$ value of $18 \mathrm{~S}$ rRNA was subtracted from that of the gene of interest to obtain a $\Delta C_{\mathrm{T}}$ value. The $\Delta C_{\mathrm{T}}$ value of an arbitrary calibrator (e.g., an untreated sample) was subtracted from the $\Delta C_{\mathrm{T}}$ value of each sample to obtain a $\Delta \Delta C_{\mathrm{T}}$ value. The gene expression level relative to the calibrator was expressed as $2^{-\Delta \Delta C_{\mathrm{T}}}$.

\section{Results}

\section{1 cDNA microarray construction}

The PCR products of 10250 unique ESTs that included 3686 contigs and 6564 singletons were spotted onto the cDNA microarrays. All the EST sequences have been submitted to GenBank and detailed information about GenBank IDs, contig assemblies, and EST annotations can be obtained from our T. rubrum database (http://www.mgc.ac.cn/ TrED/) [16].

\subsection{Transcript profiling of genes expressed during dif- ferent growth phases of $T$. rubrum}

To better understand the growth dynamics and to monitor the transcriptional expression profile during cultivation, the growth curves of $T$. rubrum were estimated by weighing the dry mass every day (Figure 1). The time points of $0,2,6$ and 10-day at different growth stages were used in the cDNA microarray analyses. In total, 2044 differentially expressed genes were obtained in these experiments. The genes were classified as involved in translation, ribosomal structure, genetic information storage and processing $(10.7 \%)$; in cell structure, cell processes and signaling $(13.6 \%)$; and in metabolism (12.18\%). However, most of the genes were classed as having "unknown function" (63.4\%). The 2044 genes were then subjected to hierarchical clustering analysis using the TIGR MultiExperiment Viewer $(\mathrm{MeV})$ to reveal the relative expression patterns of

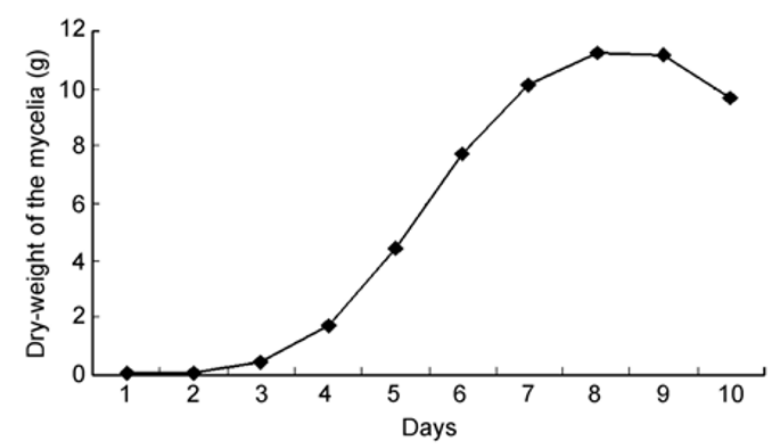

Figure 1 The growth curve of T. rubrum. T. rubrum was incubated in liquid Sabauraud medium and the dry-weight of the mycelia was measured every day. The dry-weight values from 1 to 10 days were $0.05,0.11,0.48$, $1.72,4.43,7.72,10.11,11.23,11.14$ and $9.71 \mathrm{~g}$. 
T. rubrum cultured at different growth stages [18]. Three hierarchical clusters were selected as representative clusters by visual inspection. Each of the selected clusters showed distinctive profiles (Figure 2).

Clusters I, II and III contained 733, 813 and 498 genes, respectively. The expression of Cluster I genes was reduced from time point 0 , and the lowest expression was at the last examined time point. Transcripts of genes in Cluster II increased from time point 0 and reached their peak expression at the 2-day or 6-day time point. The genes in Cluster III maintained low expression levels at the first three time points and then expression levels increased at the 6-day or 10-day time point.

The biological function and hierarchical cluster distribu-

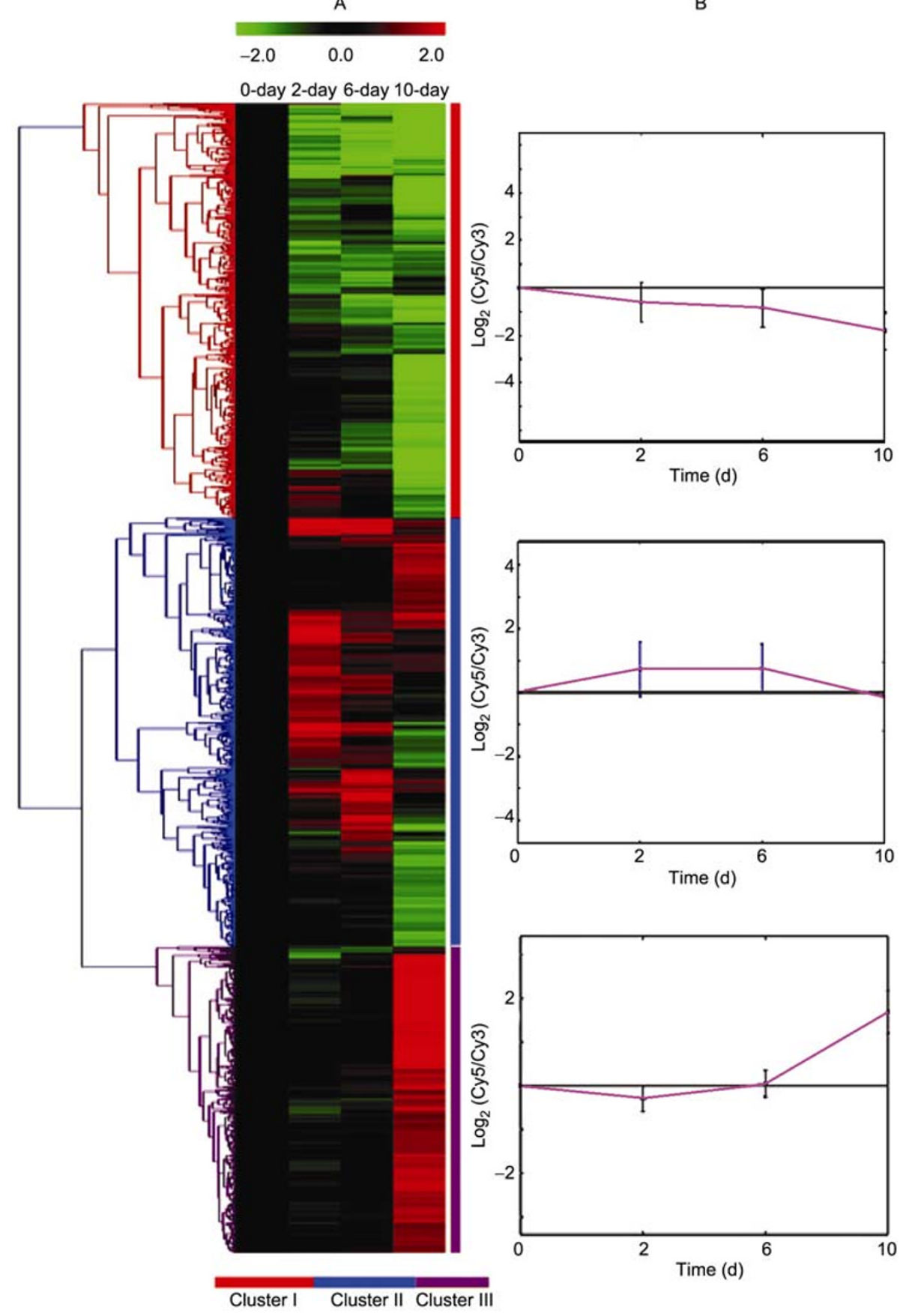

Figure 2 Hierarchical clustering of the microarray data and identification of gene expression patterns at different growth phases. A, A total of 2044 genes were clustered on the basis of their expression data from four selected time points in different growth phases using a hierarchical clustering method. The similarity in expression patterns between the genes was measured as Euclidean distance. Three distinct clusters were selected by visual inspection. The node separating each cluster is shown in the distance tree. B, An average expression profile of the genes within each cluster. C, The color code for the three se- 
tion of the genes that may be involved in the growth dynamics of T. rubrum are shown in Table 2.

\subsection{Validation of microarray data by real-time RT-PCR}

The relative expression levels of eight genes at each selected time point were estimated by quantitative real-time RT-PCR using the same RNA from the original microarray experiment. The results show a strong positive correlation between the two techniques (Table 3).

\section{Discussion}

T. rubrum is a pathogenic filamentous fungus of increasing medical concern. Here, we investigated the growth dynamics of $T$. rubrum and selected three time points in different growth stages for transcriptional profile analysis to identify genes associated with its different developmental stages. 2044 distinct $T$. rubrum genes spotted onto the microarray chips were significantly upregulated or downregulated at at least one of the four time points analyzed. To have a glob-

Table 2 The biological function and cluster distribution of the genes involved in the growth dynamics of $T$. rubrum

\begin{tabular}{|c|c|c|c|}
\hline \multirow{2}{*}{$\begin{array}{l}\text { Functional category } \\
\text { Information storage and processing }\end{array}$} & \multicolumn{3}{|c|}{ Number of genes in the cluster } \\
\hline & Cluster I & Cluster II & Cluster III \\
\hline$[\mathrm{J}]$ Translation, ribosomal structure and biogenesis & 32 & 49 & 22 \\
\hline [A] RNA processing and modification & 23 & 18 & 7 \\
\hline$[\mathrm{K}]$ Transcription & 10 & 9 & 7 \\
\hline [L] Replication, recombination and repair & 6 & 7 & 5 \\
\hline [B] Chromatin structure and dynamics & 7 & 15 & 3 \\
\hline \multicolumn{4}{|l|}{ Cellular processes and signaling } \\
\hline [D] Cell cycle control, cell division, chromosome partitioning & 7 & 10 & 8 \\
\hline$[Y]$ Nuclear structure & 3 & 4 & 1 \\
\hline$[\mathrm{T}]$ Signal transduction mechanisms & 17 & 11 & 12 \\
\hline [M] Cell wall/membrane/envelope biogenesis & 3 & 6 & 2 \\
\hline$[\mathrm{N}]$ Cell motility & 4 & 7 & 14 \\
\hline [Z] Cytoskeleton & 8 & 8 & 9 \\
\hline [W] Extracellular structures & 2 & 1 & 3 \\
\hline [U] Intracellular trafficking, secretion, and vesicular transport & 18 & 19 & 18 \\
\hline [O] Posttranslational modification, protein turnover, chaperones & 29 & 38 & 17 \\
\hline \multicolumn{4}{|l|}{ Metabolism } \\
\hline$[\mathrm{C}]$ Energy production and conversion & 11 & 29 & 8 \\
\hline [G] Carbohydrate transport and metabolism & 7 & 17 & 9 \\
\hline [E] Amino acid transport and metabolism & 23 & 22 & 7 \\
\hline$[\mathrm{F}]$ Nucleotide transport and metabolism & 7 & 6 & 6 \\
\hline$[\mathrm{H}]$ Coenzyme transport and metabolism & 3 & 6 & 5 \\
\hline [I] Lipid transport and metabolism & 19 & 18 & 18 \\
\hline$[\mathrm{P}]$ Inorganic ion transport and metabolism & 5 & 7 & 3 \\
\hline [Q] Secondary metabolites biosynthesis, transport and catabolism & 5 & 5 & 3 \\
\hline \multicolumn{4}{|l|}{ Poorly characterized } \\
\hline [R] General function prediction only & 461 & 482 & 297 \\
\hline [S] Function unknown & 23 & 19 & 14 \\
\hline Total number of genes & 733 & 813 & 498 \\
\hline
\end{tabular}

Table 3 The relative fold changes for eight genes determined by quantitative real-time RT-PCR and microarray hybridization

\begin{tabular}{|c|c|c|c|c|c|c|c|c|c|c|}
\hline \multirow{2}{*}{ Target genes } & \multirow{2}{*}{ Cluster } & \multicolumn{2}{|c|}{ 0-day } & \multicolumn{2}{|c|}{ 2-day } & \multicolumn{2}{|c|}{ 6-day } & \multicolumn{2}{|c|}{ 10-day } & \multirow{2}{*}{$r^{c)}$} \\
\hline & & $R^{\mathrm{a})}$ & $M^{\mathrm{b})}$ & $R$ & $M$ & $R$ & $M$ & $R$ & $M$ & \\
\hline DW685106 & III & 1 & 1 & 0.24 & 0.439 & 2.41 & 1.756 & 1.33 & 1.366 & 0.975 \\
\hline DW680891 & I & 1 & 1 & 0.77 & 0.573 & 1.47 & 0.801 & 0.62 & 0.336 & 0.66 \\
\hline DW679448 & II & 1 & 1 & 1.76 & 1.25 & 3.79 & 1.837 & 0.63 & 0.458 & 0.946 \\
\hline DW678242 & II & 1 & 1 & 1.54 & 1.323 & 1.12 & 1.154 & 0.49 & 0.362 & 0.963 \\
\hline DW678984 & I & 1 & 1 & 0.77 & 0.762 & 0.44 & 0.734 & 0.31 & 0.156 & 0.852 \\
\hline DW681520 & II & 1 & 1 & 3.88 & 1.673 & 0.97 & 1.000 & 0.47 & 0.429 & 0.921 \\
\hline DW691154 & III & 1 & 1 & 0.79 & 1.083 & 2.43 & 1.703 & 3.14 & 4.027 & 0.885 \\
\hline
\end{tabular}

a) $R$ is the fold change determined by quantitative real-time RT-PCR. b) $M$ is the fold change determined by microarray hybridization. c) The correlation coefficient $(r)$ for these two technologies was calculated using SPSS 13.0 software (SPSS Inc., Chicago, IL). 
ally structured view of the expression patterns of these genes, we performed a cluster analysis using a hierarchical method. The similarity in expression patterns between genes was measured as Euclidean distance.

The genes in Cluster I had the highest expression levels at time point 0 . Because dormant conidia were used in this study at time point 0 , the genes may be prestored in conidia before cultivation. The ability of fungal spores to store prepackaged mRNA has been reported in $S$. cerevisae, A. nidulans and $N$. crassa $[19,20]$. The prestored mRNA in conidia can be activated and translated rapidly in the presence of nutrients and soon after germination the stored mRNA decayed [21]. In a previous study, we found stored mRNA in T. rubrum conidia and this had similar expression characteristics to the mRNA of the Cluster I genes in this study [13].

The mRNA of genes in Cluster II was induced at the beginning of the culture process and had the highest expression levels at the 2-day and 6-day time points. The functional classification of these genes showed that a large number of them may be involved in protein biosynthesis, including genes encoding ribosomal proteins, translational initiation and elongation factors, and aminoacyl-tRNA synthetases.
Many genes related to central carbon metabolism also fall into this cluster (Table 4). For example, genes annotated as encoding glycolytic enzymes such as hexokinase (DW705801), phosphofructokinase (DW696822) and pyruvate kinase (EL789609) which are responsible for catalyzing the essentially irreversible steps in glycolysis; genes encoding the tricarboxylic acid cycle enzymes such as citrate synthase (DW704015), isocitrate dehydrogenase (DW406740), succinate dehydrogenase (DW690897, DW691173), and aconitase/homoaconitase (DW695764); genes of the electron transport system and genes related to ATP proton motive force such as cytochrome c oxidase (DW694849, DW704843) and electron transfer flavoprotein genes (DW704605, DW697873) were found in Cluster II. The glycolytic enzymes showed similar expression patterns which indicated that these enzymes in the glycolytic pathway may be coregulated.

The growth curve indicated that $T$. rubrum growth from time points 0 to the 6-day time point was in two stages, a lag phase and an exponential phase (Figure 1). A series of complex morphological and biochemical events, including conidia germination, the formation of germ tubes and proliferation of the mycelium occurred during this period. Our data suggested that the synthesis of many new proteins is

Table 4 Some genes from Cluster II involved in glycolysis, in the citrate cycle and in oxidative phosphorylation

\begin{tabular}{|c|c|c|}
\hline GenBank ID & Gene annotation & EC number \\
\hline \multicolumn{3}{|l|}{ Glycolysis } \\
\hline EL789609 & Pyruvate kinase & EC:2.7.1.40 \\
\hline DW696822 & Phosphofructokinase & EC:2.7.1.11 \\
\hline DW678636 & Zinc-binding oxidoreductase & EC:1.1.1.1 \\
\hline DW706057 & Zinc-binding oxidoreductase & EC:1.1.1.1 \\
\hline DW693239 & Acylphosphatase & EC:3.6.1.7 \\
\hline DW684723 & Aldehyde dehydrogenase & EC:1.2.1.3 \\
\hline DW705801 & Hexokinase & EC:2.7.1.1 \\
\hline DW406710 & Pyrophosphate-dependent phosphofructo-1-kinase & EC:2.7.1.11 \\
\hline \multicolumn{3}{|c|}{ Citrate cycle (TCA cycle) } \\
\hline DW690897 & Succinate dehydrogenase membrane anchor subunit and related proteins & EC:1.3.5.1 \\
\hline DW679201 & Succinate dehydrogenase membrane anchor subunit and related proteins & $\mathrm{EC}: 1.3 .5 .1$ \\
\hline DW704015 & Citrate synthase & EC:2.3.3.1 \\
\hline DW678770 & Dihydrolipoamide succinyltransferase & EC:2.3.1.61 \\
\hline DW685757 & NADP-dependent isocitrate dehydrogenase & EC:1.1.1.42 \\
\hline DW697873 & Succinate dehydrogenase, flavoprotein subunit & EC:1.3.5.1 \\
\hline DW696822 & Phosphofructokinase & EC:4.1.1.49 \\
\hline DW691173 & Succinate dehydrogenase membrane anchor subunit and related proteins & EC:1.3.5.1 \\
\hline DW695764 & Aconitase/homoaconitase & EC:4.2.1.3 \\
\hline DW406740 & Isocitrate dehydrogenase, NADP-dependent & EC:1.1.1.41 \\
\hline \multicolumn{3}{|c|}{ Oxidative phosphorylation } \\
\hline DW694849 & Cytochrome c oxidase, subunit I & EC:1.9.3.1 \\
\hline DW704843 & Cytochrome c1 & EC:1.10.2.2 \\
\hline DW405940 & Vacuolar H+-ATPase V1 sector, subunit F & EC:3.6.3.6 \\
\hline DW682879 & Vacuolar H+-ATPase V1 sector, subunit G & EC:3.6.3.6 \\
\hline DW692705 & Vacuolar H+-ATPase V0 sector, subunit a & EC:3.6.3.6 \\
\hline DW682584 & Vacuolar H+-ATPase V0 sector, subunit c" & EC:3.6.3.6 \\
\hline DW704605 & Electron transfer flavoprotein, $\beta$ subunit & EC:1.5.5.1 \\
\hline
\end{tabular}


Table 5 Genes involved in signaling transduction that were differentially expressed in response to the growth dynamics of $T$. rubrum

\begin{tabular}{|c|c|c|c|}
\hline GenBank ID & Cluster & Gene symbol & Annotation \\
\hline DW406014 & III & SAR1 & GTPase SAR1 and related small G proteins \\
\hline DW678537 & III & $\operatorname{srp} A$ & Signal recognition particle, subunit Srp54 \\
\hline DW678712 & II & & GTPase-activating protein \\
\hline DW679021 & III & Rab GTPase & Rab GTPase interacting factor \\
\hline DW679207 & II & $C D C 42$ & Ras-related small GTPase \\
\hline DW680652 & II & $Y l q F$ & mitochondrial GTPase \\
\hline DW685863 & III & GTPase $S A R 1$ & Ras-related GTPase \\
\hline DW689255 & II & Rab18 GTPase & Ras-related GTPase \\
\hline DW691671 & III & & Septin family protein (P-loop GTPase) \\
\hline DW692008 & II & Ste 7 & MAP kinase kinase (Ste7) \\
\hline DW693966 & II & & Mitofusin 1 GTPase \\
\hline DW695517 & II & & MAP kinase cascade regulation \\
\hline DW698798 & II & & Ran GTPas e-activating protein \\
\hline DW700119 & II & & GTP-binding protein (ODN superfamily) \\
\hline DW704381 & II & PKA regulatory subunit & cAMP-dependent protein kinase types I and II \\
\hline DW706608 & II & $I M E 2$ & MAPK related serine/threonine protein kinase \\
\hline
\end{tabular}

needed for germination, morphological transitions and vegetative growth. These results also indicated that during protein synthesis the energy and intermediate needs were, at least, in part provided by the degradation of glucose through glycolysis.

The further growth of $T$. rubrum finally led to one or more of the nutrients become limiting or to the accumulation to toxic levels of the metabolic by-products and 10 days after cultivation, the growth rate of $T$. rubrum declined. Most genes were repressed; however, a large group of genes of the heat shock superfamily were induced in the declining phase. These genes were all in Cluster III and included genes that code for HSP70 (DW708782, DW697080), HSP90 (DW708594), HSP90 (DW691320), and the heat shock binding protein Sti1 (DW681236). The heat shock protein superfamily is involved in response to diverse physiological stresses and has been studied in many organisms [22-24]. Our results imply that these genes may have similar biological roles in T. rubrum in its response to environmental stress.

Our data also showed that some signaling transduction pathway genes were differentially expressed in the growth dynamics of T. rubrum. Small GTPases exist as monomers that function as molecular switches in intracellular signaling to control a wide variety of cellular functions. Most of the small GTPase superfamily members have been classified into five distinct subfamilies: Ras, Rho/Rac, Rab, Arf/Sar1 and Ran. The most well-studied members are the Ras GTPases and they are sometimes called Ras superfamily GTPases. In fungi, small GTPases are important regulators of diverse cellular and developmental events including differentiation, cell division, vesicle transport, nuclear assembly, and control of the cytoskeleton [25,26]. The cAMPdependent protein kinase signaling pathway regulates growth, metabolism, environmental stress responses, entry into stationary phase, and pseudohyphal differentiation. In fungi [27] and other eukaryotic cells, MAPK cascades are used to sense changes in their environment and to transmit the signal to the nucleus to activate the proper response [28]. In our data (Table 5), changes in the expression of nine genes (DW406014, DW679021, DW679207, DW682565, DW685863, DW689255, DW698798, DW699301, and DW700119) involved in small GTPase signaling pathways were induced during the different growth phases. DW704381, a gene that encodes cAMP-dependent protein kinase types I and II and three genes, DW706608, DW695517 and DW692008, related to MAPK regulation cascades exhibited altered transcriptional levels with the growth stages transition. Although the detail biological roles of the different genes in T. rubrum are unknown, the observed differential expression that corresponded to different growth phases implied that these genes may be involved in regulatory mechanisms in the life cycle of the fungus.

This work was supported by the National Natural Science Foundation of China (Grant No. 30870104), the Eleven-Fifth Mega-Scientific Project on Infectious Diseases, China (Grant Nos. 2008ZX10401-3 and 2009ZX10004303), and an intramural grant from the Institute of Pathogen Biology, Chinese Academy of Medical Sciences (Grant No. 2006IPB008).

1 Costa M, Passos X S, Souza L K, et al. Epidemiology and etiology of dermatophytosis in Goiania, GO, Brazil. Rev Soc Bras Med Trop, 2002, 35: 19-22

2 Jennings M B, Weinberg J M, Koestenblatt E K, et al. Study of clinically suspected onychomycosis in a podiatric population. J Am Podiatr Med Assoc, 2002, 92: 327-330

3 Monod M, Jaccoud S, Zaugg C, et al. Survey of dermatophyte infections in the Lauseanne area Switzerland. Dermatology, 2002, 205: 201-203

4 Ajello L. Natural history of dermatophytes and related fungi. Mycopathologia, 1970, 53: 93-110

5 Babel D E, Rogers A L, Beneke E S. Dermatophytosis of the scalp: 
incidence, immune response, and epidemiology. Mycopathologia, 1990, 109: 69-73

6 Jackson C J, Barton R C, Glyn E, et al. Species identification and strain differentiation of dermatophyte fungi by analysis of ribosomal-DNA intergenic spacer regions. J Clin Microbiol, 1999, 37: 931-936

7 Mukherjee P K, Leidich S D, Isham N, et al. Ghannoum, clinical Trichophyton rubrum strain exhibiting primary resistance to terbi nafine. Antimicrob Agents Chemother, 2003, 47: 82-86

8 Hughes T R, Marton M J, Jones A R, et al. Functional discovery via a compendium of expression profiles. Cell, 2000, 102: 109-126

9 Giaever G, Chu A M, Ni L, et al. Functional profiling of the Saccharomyces cerevisiae genome. Nature, 2002, 418: 387-391

10 Breakspear A, Momany M. The first fifty microarray studies in filamentous fungi. Microbiology, 2007, 153: 7-15

11 Wang L L, Ma L, Leng W C, et al. Analysis of part of the Trichophyton rubrum ESTs. Sci China Ser C-Life Sci, 2004, 47: 389-395

12 Yang J, Chen L, Wang L L, et al. TrED: the Trichophyton rubrum Expression Database. BMC Genomics, 2007, 8: 250

13 Liu T, Zhang Q, Wang L L, et al. The use of global transcriptional analysis to reveal the biological and cellular events involved in distinct development phases of Trichophyton rubrum conidial germination. BMC Genomics, 2007, 8: 100

14 Yu L, Zhang W, Liu T, et al. Global gene expression of Trichophyton rubrum in response to PH11B, a novel fatty acid synthase inhibitor. J Appl Microbl, 2007, 103: 2346-2352

15 Yu L, Zhang W L, Wang L L, et al. Transcriptional profiles of the response to ketoconazole and amphotericin B in Trichophyton rubrum. Antimicrob Agents Chemother, 2007, 51: 144-153

16 Wang L L, Ma L, Leng W C, et al. Analysis of the dermatophyte Trichophyton rubrum expressed sequence tags. BMC Genomics, 2006, 7: 255
17 Hayes A, Zhang N, Wu J. Hybridization array technology coupled with chemostat culture: tools to interrogate gene expression in Saccharomyces cerevisiae. Methods, 2002, 26: 281-290

18 Saeed A I, Sharov V, White J, et al. TM4: a free, open-source system for microarray data management and analysis. Biotechniques, 2003, 342: $374-378$

19 Hollomon D W. RNA synthesis during fungal spore germination. J Gen Microbiol, 1970, 62: 75-87

20 Osherov N, May S G. The molecular mechanisms of conidial germination. FEMS Microbiol Lett, 2001, 199: 153-160

21 Brengues M, Pintard L, Lapeyre B. mRNA decay is rapidly induced after spore germination of Saccharomyces cerevisiae. J Biol Chem, 2002, 277: 40505-40512

22 Benjamin I J, McMillan D R. Stress (heat shock) proteins: molecular chaperones in cardiovascular biology and disease. Circ Res, 1998, 83: $117-132$

23 Gasch A P. Comparative genomics of the environmental stress response in ascomycete fungi. Yeast, 2007, 24: 961-976

24 Fulda S, Gorman A M, Hori O, et al. Cellular stress responses: cell survival and cell death. Int J Cell Biol, 2010, doi: 10.1155/2010/214074

25 Lundquist E A. Small GTPases. In: WormBook, ed. The C. elegans Research Community, WormBook. 2006, 17: doi/10.1895/wormbook.1.67.1, http://www.wormbook.org

26 Yoshimi T, Sasaki T, Matozaki K. Small GTP-binding proteins. Physiol Rev, 2001, 81: 153-208

27 Thevelein J M, Cauwenberg L, Colombo S, et al. Nutrient-induced signal transduction through the protein kinase A pathway and its role in the control of metabolism, stress resistance, and growth in yeast. Enzyme Microb Technol, 2000, 26: 819-825

28 Lorenz M C, Heitman J. Yeast pseudohyphal growth is regulated by GPA2, a G protein $\alpha$ homolog. EMBO J, 1997, 16: 7008-7018

Open Access This article is distributed under the terms of the Creative Commons Attribution License which permits any use, distribution, and reproduction in any medium, provided the original author(s) and source are credited. 